\title{
Performance Analysis of a Generalized and Autonomous DRX Scheme
}

\author{
Enjie Liu and Weili Ren
}

\begin{abstract}
A generalized and autonomous DRX (discontinuous Reception) scheme, applicable to both 3GPP and IEEE 802.16e standards, is analyzed by two-level Markov chain modeling along with the ETSI packet traffic model. Numerical analysis showed that this scheme is capable of autonomously adjusting DRX cycle to keep up with changing UE activity level with no signaling overhead increase, thus produces a better tuned DRX operation. Quantitative comparison with the power saving schemes of 3GPP and 802.16e standards demonstrated that it is advantageous over and generalization of these power saving schemes.
\end{abstract}

Index Terms-3G, DRX, IEEE 802.16e, LTE, LTE-Advanced, power saving, sleep mode

\section{INTRODUCTION}

$\mathrm{D}$ $\mathrm{RX}$ operation has been a dominant approach for power saving in mobile handsets. From 3GPP HSPA+ (Evolved High Speed Packet Access) and LTE (Long Term Evolution) onward, DRX operation has been introduced into the Connected State in order to save battery further during the inactive periods between packets and packet calls [1][2][3]. 3GPP RAN2 discussed over a great number of power saving proposals submitted for DRX operation in LTE/LTEAdvanced [6][7][8][9] and the finalized power saving algorithm is the short-long DRX scheme [1][2]. The DRX operation starts with the short DRX cycle and is triggered to switch to the long DRX cycle by the short DRX timer. IEEE 802.16e standards introduced the Sleep mode, and carefully considered the QoS (Quality of Service) of the supported services. Three types of power saving classes were defined for Sleep mode to fit different characteristics of diverse services. Among them, power saving class type I is dedicated for traffic connection of Best Effort and Non-Real Time Varying Rate service types and uses the exponential increase algorithm to extend sleep window size [4].

Much effort has been made to enhance these standardized power saving schemes [11][15][16][17]. In [11], it was shown

Copyright (c) 2013 IEEE. Personal use of this material is permitted. However, permission to use this material for any other purposes must be obtained from the IEEE by sending a request to pubs-permissions@ @ieee.org.

Manuscript received June 3, 2013; revised December 16, 2013 and April 6, 2014; accepted June 24, 2014.

Enjie Liu is with Department of Computer Science and Technologies, University of Bedfordshire, Park Square, Luton, Bedfordshire, UK LU1 3JU (e-mail: enjie.liu@beds.ac.uk)

Weili Ren is with BlackBerry UK, 200 Bath Road, Slough, Berkshire, UK SL1 3XE (e-mail: wren@blackberry.com) that power saving performance and packet delivery delay contradicts with each other. Trade-off can be made between them by adjusting DRX cycle, but the adjusting algorithm is yet to be studied. In [12], instead of the exponential increase algorithm standardized in 802.16e, different distributions for determining the sleep interval, such as power function algorithm were also studied. In [16], the authors proposed a statistical control approach to select the sleep window size appropriate for the present traffic pattern, which is estimated by using the number of buffered packets arriving during the previous control cycle. In [17], the authors proposed using periodic traffic indication to keep the MS in Sleep mode after data transmission, instead of using signaling exchange MOBSLP-REQ/RSP to re-enter Sleep mode. A variant of 802.16e power saving class I was proposed in [18], in which initial sleep window is dynamically tuned according to the last sleep interval before the MS wakes from sleeping. The authors in [19] proposed an adaptive power saving mechanism for 802.16e power saving class I, in which both initial sleep interval and final sleep interval are dynamically adjusted, based on the request period of previous initiation of awakening in order to maximize power saving.

These power saving schemes can efficiently operate within a certain traffic load range. To keep them operating efficiently with bursty data traffic across a wide range of time scales, the network needs to reconfigure the UE with different DRX setting whenever the UE activity level substantially changes. The reconfiguration of DRX setting would lead to a great deal of aggregating signaling overhead increase since a very large number of UEs in DRX mode are expected to be supported per cell.

We originally proposed an adaptive sleep mode scheme for 802.16e in [13] and then tailored this proposal to an adaptive DRX scheme for beyond 3G mobile handsets in [14]. The scheme ensures efficient DRX operation across a wide range of the UE activity level by using two counters $C l$ and $C 2$ in the UE and eNB respectively. The $C l$ increments when a silent DRX period occurs and resets when an active DRX period occurs, whereas the $C 2$ increments when an active DRX period occurs and resets when a silent DRX period occurs. The UE is configured with a set of DRX cycles and two DRX adjusting thresholds $N$ and $M$ by the network, based on the QoS requirements of the supported data services. When the $C 1$ (or $C 2$ ) reaches the threshold $N$ (or $M$ ), the DRX cycle is extended (or reduced) to the next (or previous) one. Since the UE and eNB have the exactly same knowledge of data 
transmissions that have happened between them, they shall adjust DRX cycle in conformity without any RRC or MAC signaling. In [13], we described how the sleep mode scheme adaptively adjusts sleep window size when user activity level changes and demonstrated by system simulation the scheme's power saving performance. In [14], we presented a simplified Markov chain model for the adaptive DRX scheme and analyzed the scheme's power saving performance and packet delay by adopting traditional Poisson traffic model.

This paper is a comprehensive extension of our research effort in [13][14]. The contributions of this paper are: 1) a more accurate and realistic analytical model of two-level Markov chains is produced for the autonomous DRX scheme the DRX state Markov chain at high level and the ActiveSleeping two-state Markov chain at low level; 2) the autonomous DRX scheme is numerically analyzed by adopting the more appropriate ETSI packet traffic model; 3) effects of thresholds $M$ and $N$ on power saving performance and packet delay are numerically computed in order for the network to set them according to different QoS requirements; 4) the autonomous DRX scheme is quantitatively compared with the power saving schemes of $3 \mathrm{GPP}$ and $802.16 \mathrm{e}$ to demonstrate that it is advantageous over and generalization of these power saving schemes; and 5) how the autonomous DRX scheme is deployed in 3GPP standards is addressed in details.

\section{ANALYTICAL MODEL FOR AUTONOMOUS DRX SCHEME}

\section{A. Markov Chain Modeling}

In the autonomous DRX scheme, the UE is configured with a set of DRX cycles $D R X_{i}, i \in(1,2, \ldots, n)$ by the network, where $D R X_{i}<D R X_{i+l}$. The UE stays in one of these DRX cycles while operating in DRX mode. Inside each of $D R X_{i}, i$ $\in(1,2, \ldots, n)$, there exist two states $S_{i}$ and $A_{i} . S_{i}$ denotes Sleeping state, in which the wake-up windows are immediately followed by sleeping, whereas $A_{i}$ denotes Active state, in which the wake-up windows are followed by data transmission then sleeping. While the UE is in $S_{i}$ state and one or more packets arrive, next wake-up window will be followed by data transmission, thus the UE will transit to $A_{i}$ state in the next DRX period. While the UE is in $A_{i}$ state and no more packets arrive after current data transmission, next wake-up window will not be followed by data transmission, thus the UE will transit to $S_{i}$ state in next DRX period.

When $S_{i}$ and $A_{i}, i \in(1,2, \ldots, n)$ are considered as the states of the autonomous DRX scheme, they form the state space of the autonomous DRX scheme and the state transitions are illustrated in Figure 1, where $p_{j, j+1}, j \in(1,2, \ldots, n-1)$ denote one-step transition probabilities from states $S_{j}$ to $S_{j+1} ; p_{k, k-1}, k$ $\in(2,3, \ldots, n)$ denote one-step transition probabilities from states $A_{k}$ to $A_{k-I} ; q_{S i, A i}$ denotes one-step transition probability from $S_{i}$ to $A_{i}$ and $q_{A i, S i}$ denotes one-step transition probability from $A_{i}$ to $S_{i}$. It is a time-homogeneous Markov chain with finite state space, and also it is irreducible and aperiodic.

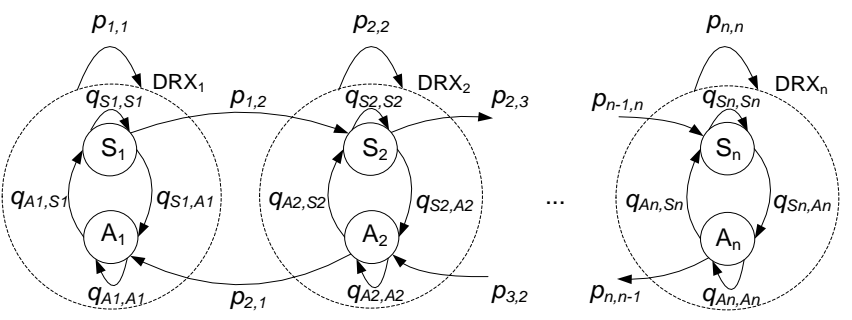

Figure 1 State transition diagram of the autonomous DRX scheme

To solve the Markov chain model, states $S_{i}$ and $A_{i}$ are combined into single state $D R X_{i}$, and the $S_{i}$ and $A_{i}$ are treated as sub-states inside state $D R X_{i}$, Therefore the Markov chain model is transformed into a new two-level Markov chain model: a Markov chain with new state space of $D R X_{i}, i \in(1,2$, $\ldots, n)$ at high level and a Markov chain with states $S_{i}$ and $A_{i}$ at low-level. Let $\Phi_{i}, i \in(1,2, \ldots, n)$ denote distribution probabilities of the UE being in states $D R X_{i}, i \in(1,2, \ldots, n)$. There always exists a unique stationary distribution $\boldsymbol{\Phi}=\left[\Phi_{l}\right.$, $\left.\Phi_{2}, \ldots, \Phi_{n}\right]$ that makes the following equation hold when equilibrium point is reached:

$\boldsymbol{\Phi}=\boldsymbol{\Phi} \cdot \boldsymbol{P}_{t}$

where $\boldsymbol{P}_{t}$ is one-step transition probability matrix, i.e.

$\boldsymbol{P}_{t}=\left[\begin{array}{cccccc}p_{1,1} & p_{1,2} & 0 & \ldots & \ldots & 0 \\ p_{2,1} & p_{2,2} & p_{2,3} & 0 & \ldots & 0 \\ \ldots & \ldots & \ldots & \ldots & \ldots & \ldots \\ \ldots & \ldots & \ldots & \ldots & \ldots & \ldots \\ 0 & \ldots & 0 & p_{n-1, n-2} & p_{n-1, n-1} & p_{n-1, n} \\ 0 & \ldots & \ldots & 0 & p_{n, n-1} & p_{n, n}\end{array}\right]$

And also

$\sum_{i=1}^{n} \Phi_{i}=1$

Existence of a unique stationary distribution ensures that the autonomous DRX scheme has steady and consistent behavior.

Inside each of states $D R X_{i}, i \in(1,2, \ldots, n)$, is the two-state Markov chain with states $S_{i}$ and $A_{i}$. Let $\Psi_{A i}$ and $\Psi_{S i}$ denote distribution probabilities of the UE being in the $A_{i}$ and $S_{i}$ respectively, then the following equation holds at equilibrium point:

$\left[\begin{array}{ll}\psi_{A i} & \psi_{S i}\end{array}\right]=\left[\begin{array}{ll}\psi_{A i} & \psi_{S i}\end{array}\right] \cdot\left[\begin{array}{cc}q_{A i, A i} & q_{A i, S i} \\ q_{S i, A i} & q_{S i, S i}\end{array}\right]$

And also

$$
\psi_{A i}+\psi_{S i}=1
$$

After $q_{A i, A i}, q_{S i, S i}, q_{A i, S i}, q_{S i, A i}$ and $\boldsymbol{P}_{\boldsymbol{t}}$ get derived, Equations (1)-(4) can be solved to get equilibrium distributions $\boldsymbol{\Psi}$ and $\boldsymbol{\Phi}$.

\section{B. Transition Probabilities}

Beyond 3G mobile networks will be data service centric, and the UE is expected to support various data services. Many of these services exhibit burstiness of data traffic across a 
wide range of time scales. Thus we adopted the ETSI packet traffic model [5], which exhibits burstiness and correlations of data traffic across a wide range of time scales, i.e. possesses long-range dependence. It has been widely used in the industry of cellular packet data communications [10]. It is assumed that the packet data traffic consists of packet service sessions. Each packet service session contains one or more packet calls depending on the applications. For example, the streaming video may comprise one single packet call for a packet session, whereas a web surfing packet session includes a sequence of packet calls. The UE initiates a packet call when requesting an information element, e.g. a WWW page. If the request is permitted, then a burst of packets will be transmitted to the UE. When the network receives the positive acknowledgement for the last packet from the UE, the current packet call transmission has completed. The time interval between the end of this packet call and the beginning of the next packet call is referred to as the inter packet call idle time $t_{i p c}$. Having received all packets of the ongoing packet service session, the UE will then experience an even longer intersession idle time $t_{i s}$. The $t_{i s}$ period represents the time interval between the end of this packet session and the beginning of the next packet session.

The statistical distributions of the ETSI packet traffic model follow the recommendation in [5] and are summarized as follows:

- The intersession idle time $t_{i s}$ is modeled as an exponentially distributed random variable with mean $1 / \lambda_{i s}$.

- The number of packet calls $N_{p c}$ within a packet service session has a geometric distribution with mean $\mu_{p c}$.

- The inter packet call idle time $t_{i p c}$ is an exponential random variable with mean $1 / \lambda_{i p c}$.

- The number of packets $N_{p}$ within a packet call follows a geometric distribution with mean $\mu_{p}$.

- The interpacket arrival time $t_{i p}$ within a packet call follows an exponential distribution with mean $1 / \lambda_{i p}$.

A packet arrival occurs in one of three cases in the ETSI packet traffic model: A) it is not the first packet of a packet call; B) it is the first packet of a packet call after inter packet call idle time; and C) it is the first packet of a packet call after intersession idle time. By using Law of total probability, the probability of no packet arriving within time duration $\tau$ is.

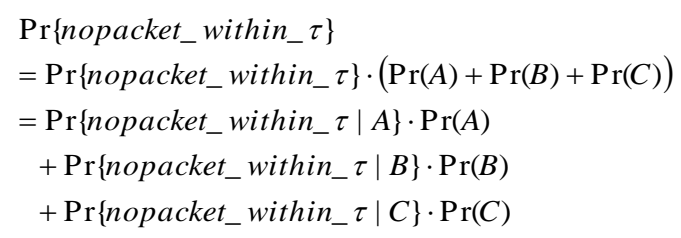

and

$$
\operatorname{Pr}(A)+\operatorname{Pr}(B)+\operatorname{Pr}(C)=1
$$

Based on the above notations for the statistical distributions of the ETSI model, we can get,

$$
\begin{aligned}
\operatorname{Pr}(A) & =\frac{T_{\text {withinpacketcalls }}}{T_{\text {withinpacktcalls }}+T_{\text {betweenpaketcalls }}+T_{\text {betweensessions }}} \\
& =\frac{\overline{N_{p c}} \cdot \overline{N_{p}} \cdot \overline{t_{i p}}}{\overline{N_{p c}} \cdot \overline{N_{p}} \cdot \overline{t_{i p}}+\overline{N_{p c}} \cdot \overline{t_{i p c}}+\overline{t_{i s}}} \\
& =\frac{\mu_{p c} \mu_{p} / \lambda_{i p}}{\mu_{p c} \mu_{p} / \lambda_{i p}+\mu_{p c} / \lambda_{i p c}+1 / \lambda_{i s}} \\
\operatorname{Pr}(B) & =\frac{T_{\text {betweenpaketcalls }}}{T_{\text {withinpacketcalls }}+T_{\text {betweenpaketcalls }}+T_{\text {betweensesions }}} \\
& =\frac{\overline{N_{p c}} \cdot \overline{t_{i p c}}}{\mu_{p c} \mu_{p} / \lambda_{i p}+\mu_{p c} / \lambda_{i p c}+1 / \lambda_{i s}} \\
& =\frac{\mu_{p c} / \lambda_{i p c}}{\mu_{p c} \mu_{p} / \lambda_{i p}+\mu_{p c} / \lambda_{i p c}+1 / \lambda_{i s}}
\end{aligned}
$$

In the case that an arriving packet is not the first packet of a packet call (Case A),

$\operatorname{Pr}\{$ nopacket_within_ $\tau \mid A\}=e^{-\lambda_{i p} \tau}$

In the case that an arriving packet is the first packet of a packet call after inter packet call idle time (Case B),

$\operatorname{Pr}\{$ nopacket_within_ $\tau \mid B\}=e^{-\lambda_{\text {ipc }} \tau}$

In the case that an arriving packet is the first packet of a packet call after intersession idle time (Case C),

$\operatorname{Pr}\{$ nopacket_within_ $\tau \mid C\}=e^{-\lambda_{i s} \tau}$

Given DRX cycles $\left(\boldsymbol{x}=\left[x_{1}, x_{2}, \ldots, x_{n}\right]\right)$, thresholds $N$ and $M$, when the UE stays in $D R X_{j}, j \in(1,2, \ldots, n-1)$, we have

$p_{j, j+1}=\left(\operatorname{Pr}\left\{\text { nopacket_within_ } x_{j}\right\}\right)^{N}$

It can be computed by using (5)-(11) for a known $x_{j}$. Similarly, for $k \in(2,3, \ldots, n)$, we have

$$
\begin{aligned}
p_{k, k-1}= & \left(\operatorname{Pr}\left\{\text { oneormore }{ }_{-} \text {within_ } x_{k}\right\}\right)^{M} \\
& =\left(1-\operatorname{Pr}\left\{\text { nopacket_within_ } x_{k}\right\}\right)^{M}
\end{aligned}
$$

For $i \in(2,3, \ldots, n-1)$

$p_{i, i}=1-p_{i, i+1}-p_{i, i-1}$

For two special cases of $p_{1,1}$ and $p_{n, n}$, we have,

$$
\begin{aligned}
& p_{1,1}=1-p_{1,2} \\
& p_{n, n}=1-p_{n, n-1}
\end{aligned}
$$

By now $\boldsymbol{P}_{t}$ has been derived, the equilibrium distribution $\boldsymbol{\Phi}$ can therefore be computed. For the low-level Markov chain with states $S_{i}$ and $A_{i}$, we have,

$$
\begin{aligned}
& q_{S i, A i}=1-\operatorname{Pr}\left\{\text { nopacket_within_ } x_{i}\right\} \\
& q_{S i, S i}=\operatorname{Pr}\left\{\text { nopacket_within_ } x_{i}\right\} \\
& q_{A i, S i}=\operatorname{Pr}\left\{\text { nopacket_within_ } x_{i}\right\}
\end{aligned}
$$


$q_{A i, A i}=1-\operatorname{Pr}\left\{\right.$ nopacket_within_ $\left.x_{i}\right\}$

They can also be computed by using (5)-(11).

\section{NUMERICAL ANALYSIS OF AUTONOMOUS DRX SCHEME}

\section{A. Power Saving and Packet Delay}

Given state of art of baseband and RF chipset technology, the UE power consumption is mainly determined by the amount of time taken while the baseband and RF circuitry is switched on. There is no definition for power saving performance in literature that can be widely adopted, therefore power waste is defined and used in the analysis of power saving performance.

Provided that wake-up window $T_{\text {wakeup }}$ is of fixed length regardless of DRX cycle. A wake-up followed by data transmission is considered no energy waste, whereas a wakeup not leading to data transmission is considered energy waste. Hence the power waste in DRX operation while the UE stays in $D R X_{i}, i \in(1,2, \ldots, n)$ can be defined as:

$$
P W_{i}=\frac{T_{\text {wakeup }}}{x_{i}} \cdot \Psi_{S_{i}}
$$

The overall power waste of the autonomous DRX scheme can be evaluated as follows:

$$
P W=\sum_{i=1}^{n} P W_{i} \cdot \Phi_{i}=\sum_{i=1}^{n} \frac{T_{\text {wakeup }}}{x_{i}} \cdot \boldsymbol{\Psi}_{S_{i}} \cdot \Phi_{i}
$$

In DRX operation, packet delivery delay occurs when packets arrive while the UE is in Sleep. Statistically the UE experiences packet delivery delay of $x_{i} / 2$ while the UE stays in $D R X_{i}, i \in(1,2, \ldots, n)$. Thus the overall packet delivery delay $D$ of the autonomous DRX scheme can be evaluated as follows:

$$
D=\sum_{i=1}^{n} D_{i} \cdot \boldsymbol{\Phi}_{i}=\sum_{i=1}^{n} \frac{x_{i}}{2} \cdot \boldsymbol{\Phi}_{i}
$$

\section{B. Numerical Analysis}

Analytical computations of power waste and packet delay have been undertaken using the Markov chain model formulated by (1)-(20). In the computations, the DRX cycles were set to be $\boldsymbol{x}=[5,10,20,40,80,160,320,640,1280$, $2560,5120] \mathrm{ms}$ and $T_{\text {wakeup }}$ was set to be $0.5 \mathrm{~ms}$. The parameters of the ETSI packet traffic model used in the computations are as follows:

$$
\begin{aligned}
& \lambda_{i p}=3.2,1.6,0.8,0.4,0.2,0.1,0.05(1 / \mathrm{ms}), \\
& \lambda_{i p c}=\lambda_{i p} / 400, \lambda_{i s}=\lambda_{i p c} / 20, \mu_{p}=25, \mu_{p c}=5
\end{aligned}
$$

Power waste and packet delivery delay of the autonomous DRX scheme were analyzed within a range of packet arrival rates, which represents varying UE traffic load at the smallest time scale. The traffic load varies further at two larger time scales (packet call and session scales) in the ETSI packet traffic model. Hence the overall traffic load appears to be very dramatic and relatively light. The numerical results are shown in Figure 2. MATLAB based system-level simulations were also undertaken under the same conditions to validate the analytical model, and the results are also shown.

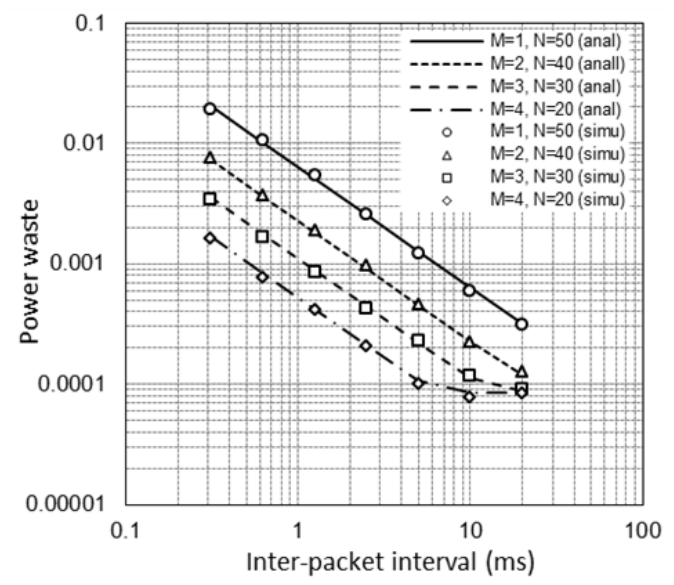

(a) Power waste

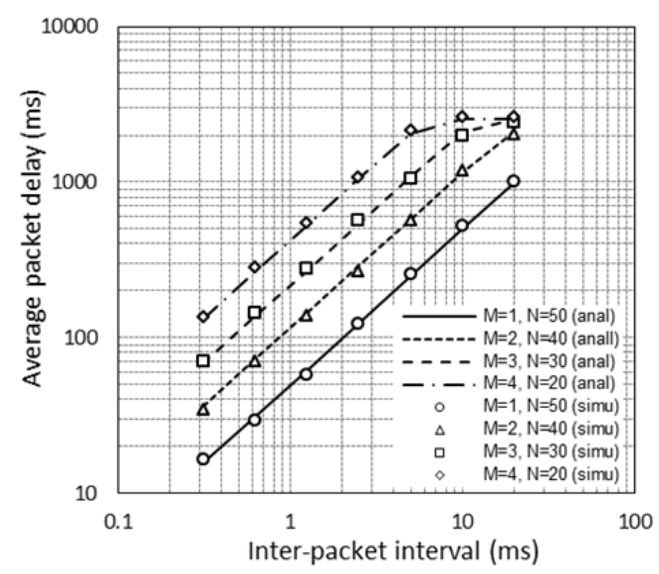

(b) Packet delay

Figure 2 Packet delay and power waste vs. inter-packet interval

Just as expected, with increasing $M$ and decreasing $N$, the autonomous DRX scheme tends to use a longer DRX cycle, resulting in longer packet delay, whereas power saving performance becomes better. With setting of $D R X_{n}=5120 \mathrm{~ms}$, packet delivery delay is capped at $D R X_{n} / 2=2560 \mathrm{~ms}$, guaranteeing maximum packet delay $<=2560 \mathrm{~ms}$. The packet delay capping occurs when the UE traffic arrives at a long inter-packet interval, meanwhile the proposed DRX scheme configures a large $M$ and a small $N$, since it leads to the UE staying in state $D R X_{n}$ (maximal DRX cycle) most of the time. When comparing packet delay and power saving in Figure 2, we can see how they contradict with each other, and how the autonomous DRX scheme can easily balance these two contradictory requirements by configuring appropriate thresholds $M$ and $N$, according to the QoS requirement of the supported data services. Contradiction of packet delay with power saving performance has shown consistently in all the numerical analyses undertaken, thus only the results of power saving performance are presented in the analysis in the following sections.

\section{Effect of Thresholds $M$ and $N$}

Threshold $M$ decides how quickly the proposed scheme reduces DRX cycle, whereas threshold $N$ decides how quickly 
the proposed scheme extends DRX cycle. They together decide how quickly the proposed scheme adjusts DRX cycle and how probable the UE stays in each of these DRX cycles for a given traffic load. For insight into the effect of thresholds $M$ and $N$ on the proposed scheme, numerical analysis was undertaken within a range of values for $M$ and $N$ respectively and analytical results are shown in Figure 3 and Figure 4.

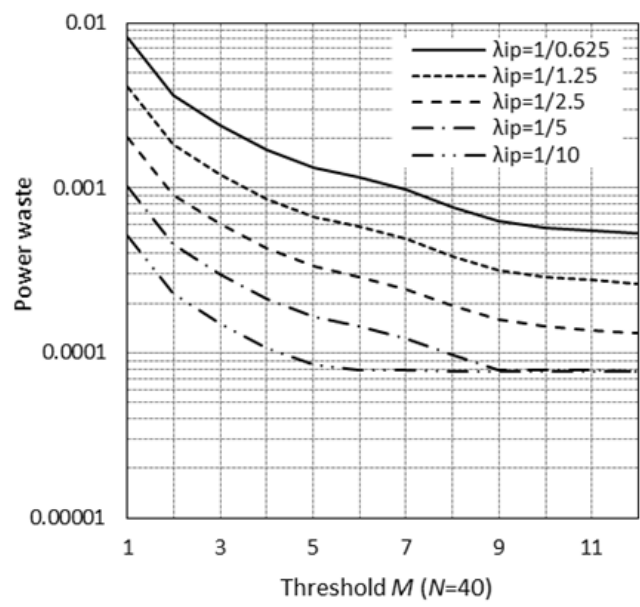

Figure 3 Effect of threshold $M$ on power waste

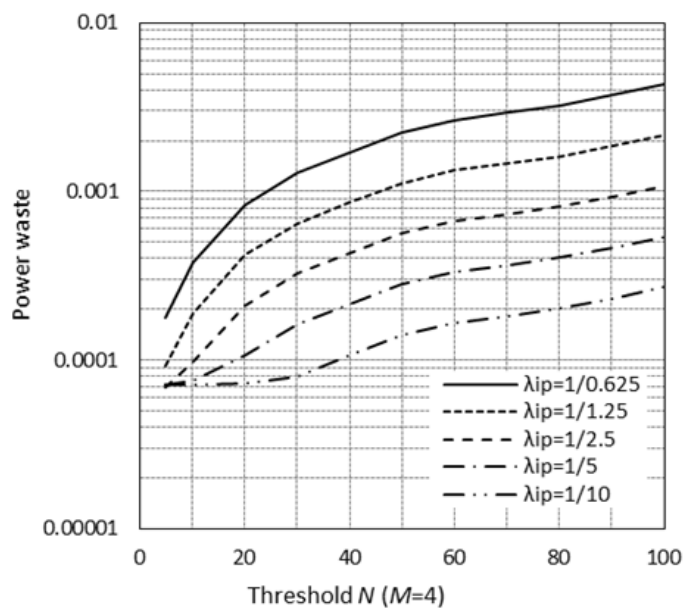

Figure 4 Effect of threshold $N$ on power waste

It can be seen that for a given traffic load, the increasing $M$ results in a decreasing power waste and an increasing average packet delay (packet delay figure is not included as explained previously), whereas the increasing $N$ results in an increasing power waste and a decreasing average packet delay. The packet delay capping occurs at $D R X_{n} / 2$ with a large $M$ or a small $N$ when traffic load is light. As expected, the analytical results also show (the figures are not included due to the limit of total number of figures) that the proposed DRX scheme presents a very similar static performance with the setting of $(M=\mathrm{a}, N=\mathrm{b})$ and the setting of $(M=2 \mathrm{a}, N=2 \mathrm{~b})$. However the scheme extends or reduces DRX cycle more quickly with $M=\mathrm{a}$ and $N=\mathrm{b}$ than with $M=2 \mathrm{a}$ and $N=2 \mathrm{~b}$, thus produces a more prompt dynamic performance.

\section{COMPARISON WITH 3GPP AND 802.16E}

\section{A. Comparison with 3GPP DRX Scheme}

The short-long DRX scheme that was standardized in 3GPP LTE/LTE-Advanced is a special case of the autonomous DRX scheme except that switch from short DRX state to long DRX state is triggered by a timer $T_{12}$. In the short-long DRX scheme, the UE switches to short DRX state right away after completing data transmission in long DRX state. The shortlong DRX scheme with typical setting of short-DRX $=20 \mathrm{~ms}$, long-DRX $=320 \mathrm{~ms}$ and $T_{12}=400,200,100$ or $40 \mathrm{~ms}$ [1] is an equivalent of the autonomous DRX scheme with setting of $D R X_{1}=20 \mathrm{~ms}, D R X_{2}=D R X_{n}=320 \mathrm{~ms}, M=1$, and $N=20$, 10,5 or 2.

Comparison analysis has been undertaken using the above Markov chain model to demonstrate the advantage of the autonomous DRX scheme over the short-long DRX scheme. In the computations, the setting of short-DRX $=20 \mathrm{~ms}$ and long-DRX $=320 \mathrm{~ms}$ was used for the short-long DRX scheme. The numerical results are shown in Figure 5, where the shortlong DRX scheme is denoted as 2DRX.

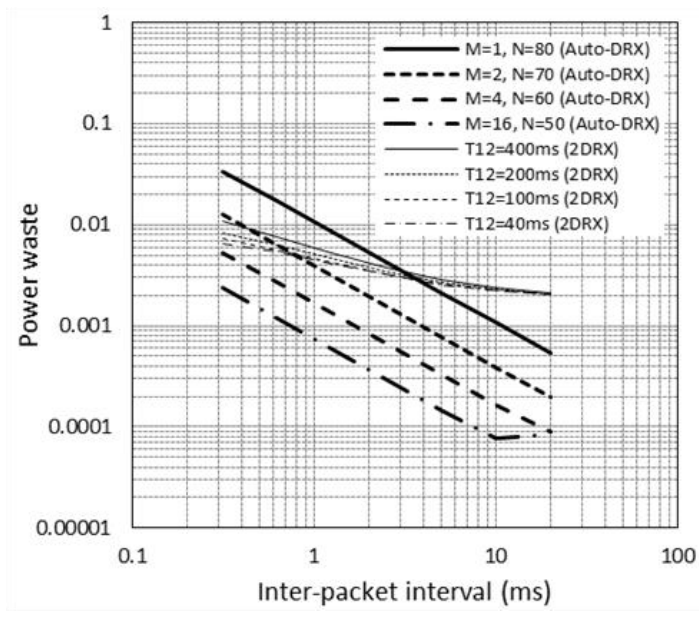

Figure 5 Comparison with short-long DRX scheme

It is clearly shown that the short-long DRX scheme cannot adapt to dramatically changing UE activity level, whereas the autonomous DRX scheme can. Furthermore, the short-long DRX scheme is not capable of balancing packet delivery delay and power saving performance. To achieve such balancing, the short-long DRX scheme has to reconfigure via RRC signaling the short-DRX and long-DRX cycles whenever the UE activity level changes substantially, which incurs a great deal of aggregating signaling overhead increase in a cell and takes up precious radio resources at the air interface.

\section{B. Comparison with IEEE 802.16e}

Comparison analysis has also been undertaken with IEEE 802.16e. To make the autonomous DRX scheme comparable with $802.16 \mathrm{e}$ power saving scheme, we selected a variant of 802.16 e power saving scheme that was initially studied in [20]. This 802.16e variant is identical to 802.16e except that the length of sleep window after an Active state reduces to previous smaller value, rather than jumps over to the smallest initial value. It operates very much like a special case of the 
autonomous DRX scheme with $M=1$ and $N=1$. The numerical results of the comparison analysis are shown in Figure 6.

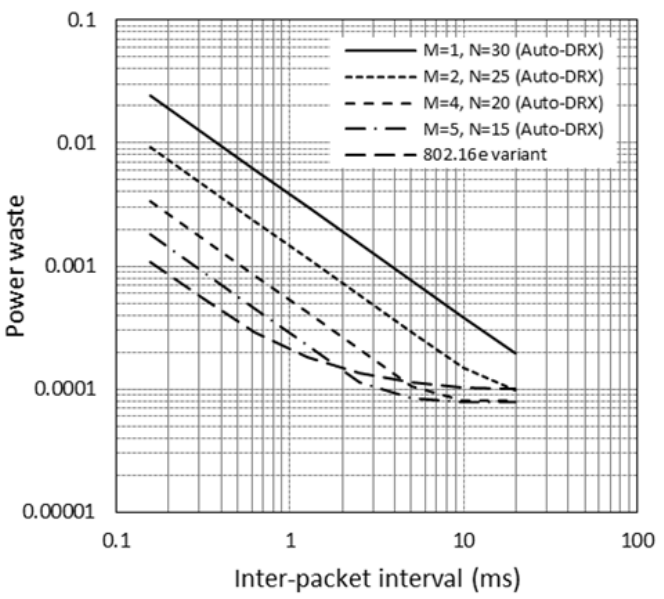

Figure 6 Comparison with an 802.16e variant

As expected, the $802.16 \mathrm{e}$ variant lacks of the ability to balance power saving performance and packet delivery delay, whereas the autonomous DRX can easily balance these two contradictory merits according to the QoS requirements of the supported data services.

\section{Adoption in 3GPP Standards}

The autonomous DRX scheme is an easy-to-implement algorithm. It is employable as an enhanced DRX feature in 3GPP HSPA+ and LTE/LTE-Advanced. Its deployment will follow the same way that other new features were added into the standards and will ensure no impact on the legacy eNBs and UEs that do not support the feature. When the feature is implemented in a UE, the UE will indicate that to the network in uplink signaling $U E$ capability information as response to downlink signaling $U E$ capability enquiry. When the feature is employed in an eNB, the network may configure a UE that supports the feature to use the feature when the UE establishes radio bearers through that eNB. The network shall not configure a UE to use the feature if the UE does not indicate that it supports the feature. The network shall not configure any UE to use the feature if these UEs establish radio bearers through an eNB that does not support the feature.

To configure the enhanced DRX feature, only does a new IE (Information Element) containing parameters $D R X_{l}, \ldots$, $D R X_{n}, M$ and $N$ need to be added into the relevant signaling messages, e.g. radio bearer setup. The eNB increments one counter and resets the other once per DRX cycle for each UE configured to use the feature. Such processing load is negligible. When one of the two counters reaches its threshold, the eNB extends or reduces DRX cycle for this UE. In contrast, when a similar traffic load change happens to a UE configured to use legacy DRX scheme, the network need reconfigure the UE with different DRX setting to adapt to the new traffic load, consequently incurring not only extra signaling overhead but also processing load. Hence adoption of the autonomous DRX scheme shall upgrade the eNB performance rather than downgrade it.

\section{CONCLUSION}

The autonomous DRX scheme was numerically analyzed by the two-level Markov chains modeling. Comparison analyses were also undertaken to demonstrate the advantage of the autonomous DRX scheme over the short-long DRX scheme of 3GPP LTE/LTE-Advanced and the power saving class type I of 802.16e. The autonomous DRX scheme is a generalized and easy-to-implement power saving scheme. It can maximize UE power saving by autonomously keeping up with the changing UE activity level, and can easily balance power saving performance and packet delivery delay, according to the QoS requirement of the data services. The short-long DRX scheme and the 802.16e sleep scheme turn out to be special cases of the autonomous DRX scheme.

\section{REFERENCES}

[1] 3GPP TS 36.331 V10.4.0, "Radio Resource Control (RRC) Protocol specification (Release 10)," 2011

[2] 3GPP TS 36.321 V10.4.0, "Medium Access Control (MAC) protocol specification (Release 10)," 2011

[3] 3GPP TS 25.331 V8.17.0, "Radio Resource Control (RRC) Protocol specification (Release 8)," 2012.

[4] IEEE 802.16e-2005, "IEEE Standard for Local and Metropolitan Area networks Part 16: Air Interface for Fixed and Mobile Broadband Wireless Access Systems," 2006.

[5] ETSI Technical Report UMTS 30.03 version 3.2.0, "Universal Mobile Telecommunications System (UMTS); Selection Procedures for the Choice of Radio Transmission Technologies of the UMTS," Apr. 1998.

[6] 3GPP R2-063397, "Views on DRX/DTX control in LTE," NTT DoCoMo

[7] 3GPP R2-063248, "DRX scheme," LG Electronics

[8] 3GPP R2-060888, "DRX and DTX operation in LTE_Active," Panasonic

[9] 3GPP R2-062819, "Adaptive DRX and DTX setting for LTE ACTIVE," Siemens

[10] S. R. Yang, S. Y. Yan, and H. N. Hung, "Modelling UMTS power saving with bursty packet data traffic," IEEE Trans. on Mobile Comp, vol. 6, no. 12, pp. 1398-1409, 2007.

[11] C. S. Bontu and E. Illidge, "DRX mechanism for power saving in LTE," IEEE Commun. Mag., vol. 47, no. 6, pp 48-55, June 2009.

[12] S. F. Lu, J. X. Wang, and Y. J. Kuang, "An efficient power saving mechanism for sleep mode in IEEE 802.16e networks," in Proc. International Wireless Commun. and Mobile Computing Conf., Caen, France, July 2010.

[13] E. Liu, J. Zhang, and W. Ren, "A counter-driven adaptive sleep mode scheme for 802.16e networks," in Proc of IEEE $73^{\text {rd }}$ Veh. Technol. Conf., May 2011.

[14] E. Liu, J. Zhang, and W. Ren, "Adaptive DRX scheme for beyond 3G mobile handsets," in Proc. IEEE Globecom, Houston, USA, Dec. 2011.

[15] T. C. Chen, J. C. Chen, and Y. Y. Chen, "Maximizing unavailability interval for energy saving in IEEE 802.16e wireless MANs," IEEE Trans. Mobile Comp., vol. 8, no. 4, pp. 475-487, Apr. 2009.

[16] C. H. Hsu, K. T. Feng, and C. J. Chang, "Statistical control approach for sleep-mode operations in IEEE $802.16 \mathrm{~m}$ systems," IEEE Tran. Veh. Technol., vol. 59, no. 9, pp. 4453-4466, Nov. 2010.

[17] E. Hwang, K. J. Kim, J. J. Son, and B. D. Choi, "The power-saving mechanism with periodic traffic indications in the IEEE $802.16 \mathrm{e} / \mathrm{m}$," IEEE Tran. Veh. Technol., vol. 59, no. 1, pp. 319-334, Jan. 2010.

[18] J. Xue, Z. Yuan, H. Chen, A. Zhang, and W. Xu, "A traffic-aware adaptive sleep mode operation for IEEE 802.16e based WiMAX," in Proc. International Conf. on Network Security, Wireless Commun. and Trusted Compu., 2009.

[19] M. G. Kim, J. Y. Choi, and M. Kang, "Adaptive power mechanism considering the request period of each initiation of awakening in the IEEE 802.16e system," IEEE Comm. Letters, vol.12, no. 2, pp. 106-108, Feb. 2008.

[20] D. G. Jeong, and W. S. Jeon, "Performance of adaptive sleep period control for wireless communications systems," IEEE Trans. Wireless Commun., vol. 5, no. 11, pp. 3012-3016, Nov. 2006. 